\title{
Phylogenetic analysis shows the general diversification pattern of deep-sea notacanthiforms (Teleostei: Elopomorpha)
}

DAVID BARROS-GARCÍA ${ }^{1,2}$ ELSA FROUFE ${ }^{3}$ RAFAEL BAÑÓN ${ }^{4,5}$, JUAN CARLOS ARRONTE $^{6}$, ALEJANDRO DE CARLOS ${ }^{1}$

${ }^{1}$ Department of Biochemistry, Genetics and Immunology, University of Vigo, C/ Fonte das Abelleiras s/n, 36310 Vigo, Spain. E-mails: davbarros@uvigo.es; adcarlos@uvigo.es ${ }^{2}$ Programa de Doctorado en Metodología y Aplicaciones en Ciencias de la Vida, Facultad de Biología. Universidad de Vigo, C/Fonte das Abelleiras s/n, 36310 Vigo, Spain.

${ }^{3}$ Centro Interdisciplinar de Investigação Marinha e Ambiental (CIIMAR/CIMAR). Terminal de Cruzeiros do Porto de Leixões, Avenida General Norton de Matos, S/N 4450-208 Matosinhos, Portugal.E-mail: elsafroufe@gmail.com

${ }^{4}$ Instituto de Investigaciones Marinas, Consejo Superior de Investigaciones Científicas (IIM-CSIC), C/ Eduardo Cabello, 6, 36208 Vigo, Spain. E-mail: anoplogaster@yahoo.es ${ }^{5}$ Grupo de Estudos do Medio Mariño (GEMM), Puerto Deportivo s/n, 15960 Ribeira, A Coruña, Spain

${ }^{6}$ Instituto de Hidráulica Ambiental (IH Cantabria). Universidad de Cantabria, PCTCAN, C/Isabel Torres nº 15, 390011, Santander, Spain. E-mail: j_arronte@ hotmail.com

Corresponding author: davbarros@uvigo.es

\section{Abstract}

The Notacanthiformes is an ancient group of deep-sea ray-finned fishes comprising 27 species in two families; Halosauridae and Notacanthidae. Although many studies have tried to reconstruct the phylogenetic relationships among the major clades of Elopomorpha, little is known about the evolutionary history of notacanthiforms. Molecular and morphological data were used to test previous hypotheses regarding the phylogenetic relationships among notacanthiform taxa, and to unravel the origin and evolution of this group. The molecular analyses of notacanthids showed similar results to those previously obtained employing osteological data, which proposed the existence of the Lipogenyinae (Lipogenys) and Notacanthinae (Notacanthus + Polyacanthonotus) subfamilies. Nevertheless, when the external morphology data is considered Lipogenys is more related to Notacanthus than Polyacanthonotus. The analyses could not fully resolve the inner relationships of the halosaurids. The time-calibrated tree of the order Notacanthiformes shows a long process of 
diversification spanning from the upper Cretaceous, to 50 million years after the K-Pg extinction, with the gradual emergence of all the modern families and genera of the group. This is the first specific phylogeny of the order Notacanthiformes, combining different analyses and data in order to obtain a wider perspective of the evolution and diversification of this group of fishes.

Keywords: Evolution; Notacanthiformes; Speciation; DNA, Bayesian

\section{Introduction}

Notacanthiformes Goodrich, 1909, is an order of deep-sea fishes most of them distributed worldwide from the continental slope to the abyssal plain (Nelson 2016). They are characterised by an eel-like body, premaxilla and maxilla bordering the upper jaw, with a connective tissue structure intercalated between perygoid and maxilla, and a posteriorly directed spine on the dorsal edge of the rear of maxilla (Nelson 2016). Despite order Notacanthiformes comprises only 27 species classified into two families, they show a wide range of feeding strategies, like hyperbenthic and detritus feeders, epifaunal browsers, and megafaunal croppers (Drazen \& Sutton, 2017, Nelson 2016).

Due to the common presence of the leptocephalus larvae, the order Notacanthiformes has been included in the superorder Elopomorpha. The latter comprises about 986 species organised in four orders, 24 families and 169 genera, and is one of the four crown teleost groups, together with Osteoglossomorpha, Otocephala and Euteleostei (Nelson et al. 2016). The phylogenetic relationships of Elopomorpha have been established with time-calibrated phylogenies, estimating the origin at about 200 million years ago, considering it the sister group of the teleosts (Broughton et al. 2013, Near et al. 2012).

60 The relationships among the four recognised orders of Elopomorpha: Albuliformes 61 (bonefishes), Notacanthiformes (halosaurs and spiny eels), Anguilliformes (eels) and 2 Elopiformes (tarpoons and ladyfishes) have been controversial historically (Chen et al. 2014). 63 Their morphology-based systematics, from the late 1960s and early 1970s, was reviewed to 64 obtain a phylogenetic analysis of 56 morphological characters (Forey et al. 1996). The results 65 showed that Elopiformes is the sister lineage that forms the earliest diverging clade of 66 elopomorphs. Later analyses based on molecular data were in accordance (Chen et al. 2014, 67 Near et al. 2012). However, in phylogenetic studies of the superorder Elopomorpha the 
notacanthiforms are represented by only a few species, without even including all recognised genera (Chen et al. 2014, Dornburg et al. 2015).

Despite the great efforts made to solve the relationships among these orders, little has been done to establish the inner relationships among notacanthiforms. A phylogeny based on osteological characters was carried out at genus level showing Lipogenys as the sister lineage of all the rest of the Notacanthidae family, proposing the creation of the subfamily Lipogenyinae (Kanehira et al. 2012). Lately, new studies based on notacanthiform species have been published: a DNA barcoding analysis highlighted the possibility of unknown taxa (Barros-García et al. 2016), in agreement with other molecular analyses that showed the possibility of cryptic species (Poulsen et al. 2017). On the other hand, an integrative taxonomy approach to North Atlantic halosaur species showed the absence of incongruities between molecular and morphological data in their systematics (Bañón et al. 2016). Therefore, in this investigation, a first assessment of the phylogenetic relationships among notacanthiforms is presented using a combination of molecular (one nuclear and three mitochondrial markers) and morphological data (combining osteological, biometric, and meristic data) in an attempt to shed light on this small but ancient branch of the tree of life. Furthermore, a time-calibrated tree clarifies the timing of notacanthiforms diversification, with ecological features offering a possible explanation.

\section{Materials and methods}

\subsection{Sample collection}

All notacanthiform individuals were morphologically identified to species level following taxonomic literature (as used in Barros-García et al. 2016). Twenty individuals representing all families and genera of the order Notacanthiformes were used (Table 1).

\subsection{Molecular data}

Genomic DNA was extracted following Barros-García et al. (2016). The three mitochondrial and one nuclear genes used for the analyses and their primers are listed in Table 2. PCR conditions were as previously described (Barros-García et al. 2016) with annealing temperatures ranging from $55^{\circ} \mathrm{C}(\mathrm{COI}, 16 \mathrm{~S}, 12 \mathrm{~S})$ to $59^{\circ} \mathrm{C}(\mathrm{ZICl})$. Sequencing was performed by Macrogen Inc., Korea using the BigDye sequencing protocol and an Applied Biosystems 3730xl genetic analyser with the same primers used in the PCR. The electrophoretograms were visually revised and edited with the software Chromas ChromasPro 2.6 (Technelysium, Tewantin, Australia). The protein-coding sequences were translated to amino acid and no stop codons were found. 
MAFFT (Kazutaka \& Standley 2016) was used to produce the individual mtDNA marker alignments, including the Anguilliformes (Notacanthifomes sister group) and the outgroup species. The former were selected and retrieved from GenBank and consisted of six species representing the order (Table 1).The outgroup consisted of Megalops cyprinoides (Broussonet 1782) and Megalops atlanticus (Valenciennes, 1847) (Elopiformes), Albula glossodonta (Forsskål, 1775) and Pterothrissus gissu (Hilgendorf 1877) (Albulidae), being Elopiformes an earlier branch of the elopomorphs, and Albuliformes a more closely-related taxa to Notacanthiformes (Kanehira et al. 2012) (Table 1). The final concatenate mtDNA data matrix was 1596 bp long and all individual sequences were deposited in GenBank (Table 1). Polymorphic positions found in ZICl corresponding to heterozygous specimens were coded according to IUPAC. The phased haplotypes were inferred probabilistically through the

112 Bayesian algorithm implemented in PHASE v2.1.1 (Stephens et al. 2001, Stephens \& Donelly, 113 2003), using SeqPHASE (Flot 2010) to prepare the input files. Software PHASE was run, under 114 default values, three times with different random seeds and checked for haplotype estimation consistency. A threshold of 0.75 posterior probability was used to accept a given haplotype phase reconstruction. All the haplotypes obtained (final length 834 bp) were submitted to GenBank (Table 1).

\subsection{Morphological data}

Three different types of data were combined in morphological analysis; osteological, biometric and meristic. The osteological data regarding notacanthiforms and both outgroups were retrieved from previous investigations (Kanehira et al. 2012; Shelyagin 2010). The material examined for biometric and meristic data is listed in Table S1. The data from Megalops and Pterothrissus were taken from Dolganov et al. 2008 and Hidaka et al. 2017. A total of 16 biometric measurements were used after being transformed relative to standard length (\% SL). Four out of the latter: preorbital, postorbital, interorbital length and eye diameter were also transformed relative to head length (\% HL), obtaining the final 20 measurements (Appendix A). Different coding strategies for the continuous characters were tested in order to obtain a correct representation of the data. The chosen strategies and the full description of all the characters used are summarized in the supplemental material (Appendix A). The final codified matrix consisted of 56 columns comprising 20 biometric, 6 meristic, and 30 osteological characters

132 (Table S2). 
For the molecular data, the best partitioning scheme was estimated with PartitionFinder2 (Lanfear et al. 2016) spanning the range from a single partition for the entire alignment to each gene and codon position treated as a partition. The saturation level of the partitions obtained was assessed with DAMBE 6 (Xia 2017) obtaining high levels of saturation for the third COI position. The most appropriate nucleotide substitution models were selected using jModelTest 2.1.8 (Darriba et al. 2012) based on the Akaike Information criterion (AIC; Akaike, 1973) for every partition. The phylogenetic trees were constructed using Bayesian Inference (BI), performed in MrBayes 3.2 (Ronquist et al. 2012). The optimal methods and the best partitioning scheme chosen are summarized in Table 2. Four Markov chains were employed in two different runs of $10 \times 10^{6}$ generations sampling every 1000 generations for a dataset of 10000 trees. No stop value was fixed and the burn-in of the first $25 \%$ of the trees was used. The software Tracer 1.6 (Rambault et al. 2014) was employed to verify the convergence of the chains and the values of the estimations of the parameters.

148 The molecular dataset, $(C O I+16 S+12 S+Z I C 1)$ including 22 sequences (2404 bp), was combined with the 56 morphological characters, totalizing 2460 positions of concatenated dataset. The BI analyses were carried out as described above using the commands: DIMENSIONS NCHART= 2460; FORMAT datatype=mixed (DNA; 1-2404, Standard: 2405-2460).

152 Divergence times of notacanthiform lineages were estimated using an uncorrelated lognormal 153 clock model in BEAST 2.4.6 (Bouckaert et al. 2014). A birth-death process was used in order to branch the tree with initial birth rate $=1.0$ and death rate $=0.5$. The molecular data was used with the nucleotide substitution models and partitions as mentioned before. Two calibration points were selected as priors for divergence time estimates, both based on Dornburg et al. 2015: most recent common ancestor (MRCA) of Elopomorpha, $\dagger$ Anaethalion zapporum resolved as a member of crown group Elopomorpha, from the Rögling formation (top of the Kimmeridgian age, dated to $152.1 \pm 09 \mathrm{Ma}$ ); MRCA Anguilliformes and Notacanthiformes, $\dagger$ Angullavis bathshebae, $\dagger$ Hayenchelys germanus resolved as stem anguilliforms, from the Hajoula Lagerstätte (Lebanese Cenomanian deposits, dated approximately 98.0 Ma). Three MCMC replicates of 20 million generations each, sampling every 1000 generations, were used.

163 Effective Sample Size (ESS) values were calculated for each parameter to check the adequate mixing of the MCMC (ESS > 200) and revised with Tracer v1.6 (Rambaut et al. 2014). Tree files from the three replicates were combined in LogCombiner v2.4.6 (Bouckaert et al. 2014) after a burn-in of $25 \%$. The Maximum Clade Credibility Tree was estimated with TreeAnnotator v. 2.4.6 (Bouckaert et al. 2014). Four different molecular datasets were used in order to test the effect of partition strategy and saturation levels in the posterior age estimates of main nodes; Partition Finder suggested partition with and without saturated positions, and all 
173 The Bayesian trees obtained from the molecular (a) and the concatenated data (b) are shown in

174 Figure 1. The obtained topologies showed the monophyly of the order Notacanthiformes, with 175 high support values in all nodes (Fig. 1a, b). In both trees, two major clusters representing the 176 Halosauridae and Notacanthidae families are recovered. The Halosauridae branch is further 177 divided in three, comprising the genera: Aldrovandia, Halosaurus and Halosauropsis, including 1786 species and 12 individuals. Although both trees recovered the monophyly of Halosauridae 179 (0.99 and 0.83 support values respectively), the relationships among these genera are poorly 180 supported $(<0.75)$ and remain unclear in both phylogenies. In the case of the Aldrovandia 181 cluster, A. affinis and A. oleosa showed a closer relationship compared to A. phalacra. On the 182 other hand, the Notacanthidae cluster showed different well supported topologies depending on 183 the data type (molecular vs concatenated), suggesting two different phylogenetic hypotheses for 184 this family. In the molecular tree (Fig. 1a) two major clusters were retrieved, the first including 185 L. gillii and the second the Notacanthus and Polyacanthonotus genera, both well supported. 186 However, the phylogeny obtained employing the concatenated data (Fig. 1b) grouped L. gillii 187 with the Notacanthus species, while P. rissoanus remained alone.

188 The estimates of split times were calculated for highly supported nodes in the final tree, inferred 189 from the optimal strategy with saturated partitions removed using BEAST v.2.4.6 (Fig. 2). The 190 tree showed the monophyly of Elopiformes and that the time estimation for the split between the 191 two species of Megalops was 26.86 mya (95\% HPD: 8.8-48.94). There is an uncertainty 192 regarding the order Albuliformes and the relationships between Albula and Pterothrissus. The 193 origin of the crown group of Anguilliformes was dated in 94 mya (95\% HPD: 72.93-114.7) 194 showing two highly supported nodes; the Anguilla + Serrivomer clade estimated in 66 mya 195 (95\% HPD: 39.67-90.47) and the node of the rest of Anguilliformes (95\% HPD: 54.25-100).

196 The analysis showed the monophyly of the order Notacanthiformes, resolving the inner 197 relationships of this taxa (BPP >0.9) with the exception of Halosauropsis, which showed 198 moderate support $(\mathrm{BPP}=0.83)$. The first split in the notacanthiform lineage happened between 199 the families Halosauridae and Notacanthidae, 73.49 mya (95\% HPD: 49.48-97.09) between the 200 second half of the Cretaceous and the early Eocene. The diversification of the Notacanthidae 201 major lineages seems to have occurred gradually from the last millions of years of the 202 Cretaceous to the early Miocene, with the Lipogenys clade separated from the rest at around 50 203 mya (95\% HPD: 29.62-72.41), and the genera Notacanthus and Polyacanthonotus splitting 20431.99 mya (95\% HPD: 16.66-49.56). Finally, the separation between N. bonaparte and $N$. 205 chemnitzii seemed to have happened at 11.31 mya (95\% HPD: 3.97-20.38). The diversification 
of the Halosauridae family, with the appearance of its major lineages, took place at the same time as Notacanthidae, appearing around 59.13 mya (95\% HPD: 38-81.81). The phylogenetic relationships among Halosauridae genera showed a moderate support to a closer relationship between Halosauropsis and Halosaurus, whereas the nodes showing the common ancestor in Aldrovandia dated 15.89 mya (95\% HPD: 6.46-27.66) and 6.98 mya (95\% HPD: 2.29-12.69) between A. affinis and A. oleosa. The split in the represented Halosaurus species, H. ovenii and H. johnsonianus, was estimated in 24.22 mya (95\% HPD: 10.69-39.49).

213 The time estimation comparisons (Fig. 2) showed more ample confidence intervals and slightly 214 more recent time estimates for those analyses without saturated partitions. For the crown node 215 of Notacanthiformes the values varied from 73.2 mya (95\% HPD: 47.8-99.5) and 73.5 (95\% 216 HPD: 49.5-97) from the datasets without saturated partitions to 76.2 (95\% HPD: 57.3-96.1) and 21779.5 (95\% HPD: 56.6-103.6) when the saturated partitions were taken into account. Similar 218 results were obtained for the Halosauridae and Notacanthidae families. In the former, the 219 analysis without saturated partitions showed an estimation of 59 mya (95\% HPD: 38-81.8) in 220 partitioned data, and 61.7 (95\% HPD: 36.6-85.1) in the concatenated data, whereas saturated partitions retrieved values of 64.2 (95\% HPD: 43.8-86) and 62.3 (95\% HPD: 44.6-81.4) respectively. The crown node for the Notacanthidae family showed similar estimations for nonsaturated data, 49 mya (95\% HPD: 27.3-72.7) and 50.2 (95\% HPD: 29.6-72.4), and when saturated partitions were used, 54.2 mya (95\% HPD: 37.4-72.2) and 57 (95\% HPD: 37.7-77.7), respectively.

\section{Discussion}

The phylogenies obtained in this investigation include representatives of all known genera of notacanthiforms and show their monophyletic nature at several taxonomical levels (order, family and genus). These results agree with those obtained in previous phylogenetic studies where notacanthiforms were partially represented among a huge number of fish taxa (Betancur et al. 2017; Chen et al. 2014; Dornburg et al. 2015).

233 However, the open question about the evolutionary relationships among Halosauridae genera Aldrovandia, Halosauropsis and Halosaurus remain unsolved due to the partial loss of node support among them, a fact also previously observed (Chen et al. 2014). The diversity of data, including mitochondrial and nuclear markers and morphological traits, may suggests the possibility of three simultaneous speciation events from the same common ancestor, a so-called "hard" polytomy.

239 The relationships among the three major lineages in the Notacanthidae family showed two 240 different hypotheses depending on the type of data used. First, the molecular data suggests 
241 Lipogenys as the sister lineage of Notacanthus and Polyacanthonotus, as previously reported in 242 an osteological-based phylogeny. There, the Lipogenys clade is supported by two osteological 243 apomorphies and the Notacanthus and Polyacanthonotus lineage by three synapomorphies 244 (Kanehira et al. 2012). Distinctly, the concatenated data show Polyacanthonotus as the sister 245 lineage of Lipogenys and Notacanthus. Because the molecular analyses arrive to the same 246 conclusion as the osteological ones, the only explanation for this discrepancy is the addition of 247 new biomeristic traits to the already existing osteological data. This would suggest that the 248 external morphology of Lipogenys is more similar to Notacanthus as they share similar 249 ecological strategies feeding on benthic organic matter or sessile organisms, while 250 Polyacanthonotus are active predators of crustaceans more than a shared common ancestor 251 (Romeu et al. 2016; Templeman, 1973). Therefore, the combination of the molecular and 252 osteological data supports the subfamily Lipogenyinae as the sister lineage of Notacanthinae, as 253 previously proposed (Kanehira et al. 2012).

254 The time calibrated analyses has dated the diversification of the notacanthiforms between the upper Cretaceous and early Eocene (95\% HPD: 49.48-97.09), a younger estimation than others 256 previously obtained (Dornburg et al. 2015, Santini et al. 2013). This difference could be related 257 to the increase in the number of specific taxa employed, which may have improved the 258 phylogenetic inference (Dornburg et al. 2015). In this period three main events happened: the $259 \mathrm{~K} / \mathrm{Pg}$ extinction (65 mya) and two different diversifications for actinopterygians; the 260 Cenomanian (about 100 mya) and the Paleocene-Eocene interval (66-33.9 mya), both the two 261 most exceptional radiations among vertebrates (Guinot \& Cavin, 2016). However, the broad 262 confidence interval does not allow us to stablish a direct relationship among any of them and the origin of notacanthiforms. Nevertheless, the fossil record of halosaurs shows that their typical morphology was already present in the upper Cretaceous (89.3-85.8 mya), suggesting that the split between Halosauridae and Notacanthidae happened before the K/Pg extinction (Dornburg et al. 2015). The confidence intervals dating the diversification of modern notacanthiform families span from the upper Cretaceous to the early Oligocene, partially overlapping with each other and with the crown node of the notacanthiforms. Unlikely, the time estimation of Halosauridae showed older values than others previously obtained (Dornburg et al. 2015). Our study has obtained for the first time a complete genus level timetree for the Notacanthidae family. The results also support the subfamily Lipogenyinae as the sister lineage of Notacanthinae, as previously proposed (Kanehira et al. 2012).

273 Despite the wide time intervals obtained, our results show a higher effect of saturation than the 274 partition strategy in the time estimation, with older estimations when saturated partitions are 275 included in the analysis. When saturation is not taken into account, the use of mitochondrial markers led to produce older ages than the nuclear ones (Dornburg et al. 2014). 
277 The order Notacanthiformes shows a long process of diversification spanning from the upper

278 Cretaceous, about 100 million years ago, to 50 million years after the K-Pg extinction, with the

279 gradual emergence of all the modern families and genera of the group. This study represents the

280 first specific phylogeny of notacanthiforms, combining different analyses and data to obtain a

281 wide perspective of the evolution and diversification of this old group of fishes. We provide

282 strongly supported results showing Lipogenys as the sister lineage of the rest of the

283 notacanthidae genera, although we cannot fully resolve the inner relationships of the

284 Halosauridae family. Future analyses, probably including extinct lineages, will be required in

285 order to achieve a complete phylogenetic hypothesis of the order Notacanthiformes and resolve

286 this branch of the Tree of Life.

\section{Acknowledgements}

289 We thank the reviewers for the helpful remarks and suggestions that improved the quality of the 290 manuscript. This investigation was partially founded by the Spanish Ministry of Environment 291 (research project ECOMARG 3), by the EC LIFE+ 'Nature Biodiversity' INDEMARES project 292 (07/NAT/E/000732) and received additional funding from the Research Line BlueSensors, 293 integrated in the Structured Program of R\&D\&I CORAL - Sustainable Ocean Exploitation: 294 Tools and Sensors (reference NORTE-01-0145-FEDER-000036), funded by the Northern 295 Regional Operational Programme (NORTE2020) through the European Regional Development 296 Fund (ERDF). EF was supported by the Portuguese Foundation for Science and Technology 297 (FCT) under grant SFRH/BPD/108445/2015. The Northwestern Atlantic notacanthiform 298 specimens were sampled during the oceanographic surveys carried out by the Instituto Español 299 de Oceanografía, C.O de Vigo through the "Programa de Pesquerías Lejanas". The authors wish 300 to thank all the participants in the surveys 'INDEMARES 0811' and the crew of the R/V 301 Miguel Oliver (Ministerio de Agricultura, Alimentación y Medio Ambiente). We are grateful to 302 Miguel Angel Puerto and Ana Ramos, both participants in the 'PLATUXA 2012' and 'CCLME 303 2012' surveys, respectively, for collecting some specimens from Canadian waters. We extend 304 our thanks also to the full research team. The Regional Government of Galicia (Xunta de 305 Galicia) has collaborated in this research through cofounding from the European Regional 306 Development Fund (ERDF). Thanks to Raymond Simpson (University of Yale) for the 307 illustrations of Halosauridae species used in the figure 2. This research paper partially fulfils the $308 \mathrm{PhD}$ degree requirements of DBG in the University of Vigo. 
314 Figure 1:

315 Phylogenetic trees constructed using the BI method. a) tree based on the molecular dataset $316(\mathrm{COI}+16 \mathrm{~S}+12 \mathrm{~S}+\mathrm{ZIC} 1)$. b) tree based on the concatenated dataset (molecular dataset + 317 morphological matrix). Support values for each node are included (values $<0.75$ are not 318 shown).

319 Figure 2:

320 Time-calibrated phylogeny of notacanthiforms based on one nuclear and three mitochondrial 321 markers and two temporal constraints. Bars represent the posterior distribution of divergencetime estimates. Black dots represent node supports $\geq 0.95$. Red dots show calibration points in the tree. The time-calibrated tree is scaled in millions of years. The red line shows the Cretaceous/Paleogene extinction event and the green areas the known actinopterygian diversification events. Illustrations were obtained from Raymond Simpson (http://watlfish.com/) and the NOAA Photo Library for Halosauridae and Notacanthidae families respectively. Inset: Comparison of ages estimated for order Notacanthiformes, Halosauridae and Notacanthidae families; Red, PartitionFinder partition with saturated positions. Blue, PartitionFinder partition without saturated positions. Violet, all concatenated with saturated positions. White, all concatenated without saturated positions

Bibliography:

- Akaike, H. 1973. Information theory as an extension of the maximum likelihood principle. In: Petrov, B.N., Csaki, F. (Eds.) Second International Symposium on Information Theory. Akademiai Kiado, Budapest.

- Barros-García, D., Bañón, R., Arronte, J.C., Fernández-Peralta, L., García, R., de Carlos, A. 2016. DNA Barcoding of deep-water notacanthiform fishes. Zool. Scr. 45 (3), 263-272.

- Bañón, R. Arronte, J.C., Armesto, A., Barros-García, D., de Carlos, A. 2016. Halosaur fishes (Notacanthiformes:Halosauridae) from Atlantic Spanish waters according to integrative taxonomy. Zootaxa. 4184, (3): 471-490. 
- Betancur, R.R., Wiley, E. D., Arratia, G., Acero, A., Bailly, N., Miya, M., Lecointre, G., Ortí G. 2017. Phylogenetic classification of bony fishes. BMC Evol. Biol. 17: 162.

- Bouckaert, R., Heled, J., Kühnert, D., Vaughan, T., Wu, C-H., Xie, D., Suchard, MA., Rambaut, A., \& Drummond, A. J. 2014. BEAST 2: A Software Platform for Bayesian Evolutionary Analysis. PLoS Comput. Biol. 10(4), e1003537. doi:10.1371/journal.pcbi.1003537

- Broughton, E.R., Betancur-R.R, Li, C., Arratia, G., Ortí, G. 2013. Multi-locus phylogenetic analysis reveals the pattern and tempo of bony fish evolution. PLoS Currents. art. no. ecurrents.tol.2ca8041495ffafd0c92756e75247483e Elopomoprha (Teleostei): Evidence from six nuclear and mitochondrial markers. Mol. Phylogenet. Evol. 70, 152-161.

- Darriba, D., Taboada, G.L., Doallo R, Posada, D. 2012. jModelTest 2: more models, new heuristic and parallel computing. Nat. Methods. 9 (8), 772.

- Dolganov, V.N., Kharin, V.E., Zemnukhov, V.V. 2008. The Megalopidae, a New Family of Fishes for Fauna of Russia. J. Ichthyol. 48, 275-276.

- Dornburg, A, Townsend, J. P., Friedman, M., Near, T.J. 2014. Phylogenetic informativeness reconciles ray-finned fish molecular divergence times. BMC EVOL. BIOL. 14, 169.

- Dornburg, A., Friedman, M.. Near, T.J. 2015. Phylogenetic analysis of molecular and morphological data highlights uncertainty in the relationships of fossil and living species of Elopomorpha (Actinopterygii: Teleostei). Mol. Phylogenet. Evol. 89, 205218.

- Drazen, J.C. \& Sutton, T. 2017. Dining in the Deep: The Feeding Ecology of Deep-Sea Fishes. Annu. Rev. Mar. Sci. 9, 337-366.

- Forey, P.L., et al. 1996. Interrelationships of Elopomorph Fishes, in: Stiassny M.L.J., Parenti, L.R., Johnson G.D. (Eds.) Interrelationships of Fishes. Academic Press., San Diego, pp. 175-190.

- Flot, J.F. 2010. Seqphase: a web tool for interconverting phase input/output files and fasta sequence alignments. Mol. Ecol. Res. 10, 162-166.

- Guinot, G. \& Cavin, L. 2016. "Fish" (Actinopterygii and Elasmobranchii) diversification patterns through deep time. 2016. Biol. Rev. 91, 950-981.

- Hidaka, K., Tsukamoto, Y., Iwatsuki, Y. 2017. Nemoosis, a new genus for the eastern Atlantic long-fin bonefish Pterotrhrissus belloci Cadenat 1937, and a redescription of P. gissu Hilgendorf 1877 from the northwestern Pacific. Ichthyol. Res. 64, 45-53.

- Ivanova, N., Zemlak, T. S., Hanner, R. H. \& Hebert, P. D. N. 2007. Universal primer cocktails for fish DNA barcoding. Mol. Ecol. Notes. 7, 544-548. 
- Kanehira, N., Imamura, H., Yabe, M. 2012. Phylogenetic relationships of the suborder Notacanthoidei (Teleostei: Albuliformes) reassessed from osteological characters, with a proposed new classification. Mem. Grad. Sci. Fish. Sci. Hokkaido Univ. 54 (1/2).

- Kazutaka, K. Standley, M. D. 2016. A simple method to control over-alignment in the MAFFT multiple sequence alignment program. Bioinformatics. 32 (13), 1933-1942.

- Lanfear, R., Frandsen, P. B., Wright, A. M., Senfeld, T., Calcott, B. 2016. PartitionFinder 2: new methods for selecting partitioned models of evolution for molecular and morphological phylogenetic analyses. Mol. Biol. Evol. 3, 772-773.

- Li, C., Ortí, G., Zhang, G., Lu, GQ. 2007. A practical approach to phylogenomics: The phylogeny of ray-finned fish (Actinopterygii) as a case study. BMC Evol Biol. 7:44.

- Near, J. T. et al. 2012. Resolution of ray-finned fish phylogeny and timing of diversification. PNAS. 119, 13698-13703.

- Nelson, J. S., Grande, T.C.,Wilson, M.V.H. 2016. Fishes of the world, 5th edn. John Wiley \& Sons, Hoboken, New Jersey.

- John Wiley \& Sons, Hoboken, New Jersey. Palumbi, S.R., Martin, A.P., Romano, S., McMillan, W.O., Stice, L., Grabowski, G. 1991. The Simple Fool's Guide to PCR, Version 2.0. Published by the authors, Honolulu.

- Poulsen, J. Y., Thorkildsen, S., Arboe, N. H. 2017. Identification keys to halosaurs and notacanthids (Notacanthiformes, Elopomorpha) in the subartic Atlantic Ocean including three new distributional records and multiple molecular OTUs of Notacanthus cf. chemnitzii. Mar. Biodiv. DOI: 10.1007/s12526-017-0762-8

- Rambault, A., Suchard, M.A., Xie, D., Drummond, A.J. 2014. Tracer v1.6 Computer Program and Documentation Distributed by the Author http://tree.bio.ed.ac.uk/software/tracer/

- Romeu, O. R., Cartes, J. E., Solé, M., Carrasón, M. 2016. To what extent can specialized species succeed in the deep sea? The biology and trophic ecology of deepsea spiny eels (Notacanthidae) in the Mediterranean Sea. Deep-Sea Res. 115, 74-90.

- Ronquist, F., Teslenko, M., van der Mark, P., Ayres, D.L., Darling, A., Hohna, S., Larget, B., Liu, L., Suchard, M.A., Huelsenbeck, J.P. 2012. MrBayes 3.2: effective Bayesian phylogenetic inference and model choice across a large model space. Syst. Biol. 61, 539-542.

- Santini, F., Kong, X., Sorenson, L., Carnevale, G., Mehta, R.S. 2013. A multi-locus molecular timescale for the origin and diversification of eels (Order: Anguilliformes). Mol. Phylogenet. Evol. 69, 884-894.

- Shelyagin, D.I. 2010. Osteology of Halosauridae (Notacanthiformes). J. Ichthyol. 50, 512-528. 
- Stephens, M., Smith, N., Donnelly, P., 2001. A new statistical method for haplotype reconstruction from population data. Am. J. Hum. Genet. 68, 978-989.

- Stephens, M., Donnelly, P., 2003. A comparison of Bayesian methods for haplotype reconstruction from population genotype data. Am. J. Hum. Genet. 73, 1162-1169.

- Templeman, W., 1973. Description and distribution of new specimens of the fish Lipogenys gilli from Western North Atlantic. J. Can. Fish. Res. Board. 30, (10): 15591564 .

- Wang, H.Y., Tsai, M.P., Tu, M.C., Lee, S.C. 2000. Universal Primers for Amplification of the Complete Mitochondrial 12S rRNA Gene in Vertebrates. Zool. Stud. 39, (1): 6166.

- Xia, X. 2017. DAMBE6: New tools for microbial genomics, phylogenetics and 427

\section{Table 1}

433 List of specimens examined in this study and GenBank accession numbers for ZIC1 and 434 mitochondrial genes (12S, 16S, COI) collected.

\begin{tabular}{|c|c|c|c|c|c|c|c|}
\hline Order & Family & Species & ID & $12 \mathrm{~S}$ & $16 \mathrm{~S}$ & $\mathrm{COI}$ & ZIC1 \\
\hline Albuliformes & Albulidae & Albula glossodonta & $\mathrm{NA}$ & NC_005800.1 & NC_005800.1 & NC_005800.1 & JX191248.1 \\
\hline Albuliformes & Albulidae & Pterothrissus gissu & NA & NC_005796.1 & NC_005796.1 & NC_005796.1 & KJ910156.1 \\
\hline Anguilliformes & Anguillidae & Anguilla rostrata & NA & KJ564206.1 & KJ564206.1 & KJ564206.1 & EU001889.1 \\
\hline Anguilliformes & Congridae & Conger japonicus & NA & NC_027186.1 & NC_027186.1 & NC_027186.1 & JX255601.1 \\
\hline Anguilliformes & Congridae & Heteroconger hassi & NA & NC_013629.1 & NC_013629.1 & NC_013629.1 & JX191254.1 \\
\hline Anguilliformes & Derichthyidae & Derichthys serpentinus & NA & NC_013611.1 & NC_013611.1 & NC_013611.1 & FJ906633.1 \\
\hline Anguilliformes & Ophichthidae & Myrichthys maculosus & NA & NC_013635.1 & NC_013635.1 & NC_013635.1 & NA \\
\hline Anguilliformes & Serrivomeridae & Serrivomer beanii & NA & NC_013627.1 & NC_013627.1 & NC_013627.1 & FJ906634.1 \\
\hline Elopiformes & Megalopidae & Megalops atlanticus & NA & NC_005804.1 & NC_005804.1 & NC_005804.1 & JX191251.1 \\
\hline Elopiformes & Megalopidae & Megalops cyprinoides & NA & NC_005799 & NC_005799 & NC_005799 & KJ910161 \\
\hline Notacanthiformes & Halosauridae & Aldrovandia affinis & NHA001 & MF156145 & MF156165 & KP845174 & MF156197 \\
\hline Notacanthiformes & Halosauridae & Aldrovandia affinis & NHA002 & MF156146 & MF156166 & KP845175 & MF156198 \\
\hline Notacanthiformes & Halosauridae & Aldrovandia oleosa & NHO001 & MF156147 & MF156167 & KP845185 & MF156191/MF156192 \\
\hline Notacanthiformes & Halosauridae & Aldrovandia oleosa & NHO002 & MF156148 & MF156168 & KP845184 & MF156193 \\
\hline Notacanthiformes & Halosauridae & Aldrovandia phalacra & JBD001 & MF156157 & MF156177 & KP845193 & MF156194/MF156195 \\
\hline Notacanthiformes & Halosauridae & Aldrovandia phalacra & JBD002 & MF156158 & MF156178 & KP845194 & MF156196 \\
\hline Notacanthiformes & Halosauridae & $\begin{array}{l}\text { Halosauropsis } \\
\text { macrochir }\end{array}$ & NHH001 & MF156141 & MF156161 & KP845201 & MF156184 \\
\hline
\end{tabular}




\begin{tabular}{|c|c|c|c|c|c|c|c|}
\hline Notacanthiformes & Halosauridae & $\begin{array}{l}\text { Halosauropsis } \\
\text { macrochir }\end{array}$ & NHH002 & MF156142 & MF156162 & KP845196 & MF156185 \\
\hline Notacanthiformes & Halosauridae & $\begin{array}{l}\text { Halosaurus } \\
\text { johnsonianus }\end{array}$ & NHI001 & MF156149 & MF156169 & KP845203 & MF156190 \\
\hline Notacanthiformes & Halosauridae & $\begin{array}{l}\text { Halosaurus } \\
\text { johnsonianus }\end{array}$ & NHIO02 & MF156150 & MF156170 & KP845204 & NA \\
\hline Notacanthiformes & Halosauridae & Halosaurus ovenii & NHU001 & MF156143 & MF156163 & KP845208 & MF156186/MF156187 \\
\hline Notacanthiformes & Halosauridae & Halosaurus ovenii & NHU002 & MF156144 & MF156164 & KP845209 & MF156188/MF156189 \\
\hline Notacanthiformes & Notacanthidae & Lipogenys gillii & TSN001 & MF156151 & MF156171 & KP845211 & MF156199 \\
\hline Notacanthiformes & Notacanthidae & Lipogenys gillii & TSN002 & MF156152 & MF156172 & MF156183 & MF156200 \\
\hline Notacanthiformes & Notacanthidae & $\begin{array}{l}\text { Notacanthus } \\
\text { bonaparte }\end{array}$ & NBO001 & MF156153 & MF156173 & KP845212 & MF156201/MF156202 \\
\hline Notacanthiformes & Notacanthidae & $\begin{array}{l}\text { Notacanthus } \\
\text { bonaparte }\end{array}$ & NBO002 & MF156154 & MF156174 & KP845234 & MF156203 \\
\hline Notacanthiformes & Notacanthidae & $\begin{array}{l}\text { Notacanthus } \\
\text { chemnitzii }\end{array}$ & NNN001 & MF156159 & MF156179 & MF156181 & MF156204 \\
\hline Notacanthiformes & Notacanthidae & $\begin{array}{l}\text { Notacanthus } \\
\text { chemnitzii }\end{array}$ & NNN002 & MF156160 & MF156180 & MF156182 & MF156205 \\
\hline Notacanthiformes & Notacanthidae & $\begin{array}{l}\text { Polyacanthonotus } \\
\text { rissoanus }\end{array}$ & NNP001 & MF156155 & MF156175 & KP845235 & MF156206/MF156207 \\
\hline Notacanthiformes & Notacanthidae & $\begin{array}{l}\text { Polyacanthonotus } \\
\text { rissoanus }\end{array}$ & NNP002 & MF156156 & MF156176 & KP845236 & MF156208 \\
\hline
\end{tabular}

\section{5}

436 Table 2

437 Information regarding molecular data. Different markers used with their respective primers.

438 Partitions selected with PartitionFinder and substitution models obtained with jModelTest.

\begin{tabular}{cccccc}
\hline Gene & Primers & Expected Size & Reference & Partition & Model \\
\hline $12 S$ & 12SL/12SH & $400 \mathrm{bp}$ & Wang et al. 2000 & $12 \mathrm{~S}$ & GTR+G+I \\
$16 S$ & 16Sar-5'16Sbr-3' & $600 \mathrm{bp}$ & Palumbi 1996 & $16 \mathrm{~S}$ & GTR+G+I \\
COI & C_FishF1t1-C_FishR1t1 & $700 \mathrm{bp}$ & Ivanova et al. 2007 & COI_1 & GTR+G+I \\
& & & & COI_2 & JC \\
& & & & COI_3 & GTR+G \\
ZIC1 & ZIC1_F9,ZZIC1_R967 & \multirow{2}{*}{$850 \mathrm{bp}$} & Li et al. 2007 & ZIC1_1 & GTR \\
& ZIC1_F16 ZIC1_R963 & & & ZIC1_2 & HKY \\
& & & & ZIC1_3 & HKY+I \\
\hline
\end{tabular}

439

440 